erwähnten direkten Methode gering. Der Vorteil der vereinfachten Auswertungsmethode besteht darin, dal unter Verzicht auf alle Berechnungen allein durch die Inspektion der Kurven eine Bestimmung des Grades der Abweichung von der Normalkurve erfolgen kann. Wenn die einzelnen Kurvenbilder für sich allein die Diagnose eines bestimmten Herzfehlers oder -leidens nicht gestatten, so erlauben sie doch eine $\mathrm{Ab}$ schätzung - keine Messung - der Herzkraft (im physikalischen Sinne). An Hand mehrerer Beispiele aus der Klinik wird gezeigt, wie sich während der Behandlung analog zur subjektiven und klinisch-objektiven Besserung des Zustandes der Patienten die pathologisch veränderten EKG-Kurven norznalisieren, während die ebenfalls abnormen EKG.Kurven im gleichen Zeitraum keine Tendenz zur Besserung erkennen ließen. An Hand weiterer Beispiele aus der Inneren Medizin, der Chirurgie und der Frauenheilkunde wird die Bedeutung der Methode für viele Zweige der Medizin unterstrichen. Dabei wird besonders auf die Anderungen der Kurven nach operativer Behandlung der Herz- und Gefäßanomalien hingewiesen. Bei einer kritischen Anwendung erweist sich die Ballistokardiographie als gutes Hilfsmittel für die Diagnose, Prognose und Beurteilung eines Therapieerfolges bei den Herz- und GefäBkrankheiten.

Fraser.

\section{Gesellschaft für Biophysik Göttingen.} Sitzung vom 14. Mai 1954.

MÜLLER, J.H.: Grumdlagen und pralktische Aussichten der Tumortherapie mit Hilfe der künstlichen Radioaktivität. Die künstlich rudioaktiven Isotope haben bereits eine ins Gewicht fallende praktische Bedeutung für die Therapie der Geschwulstkrankheiten erlangt. Bei der internen Anwendung solcher Isotope wird man nur in seltenen Ausnahmefăllen einen diffusen Effekt anstreben. In der Regel ist eine möglichst intensive Lokalisierung der radioaktiven Isotope im Bereiche der erkrankten Gewebe, Organe oder Körperregionen unbedingt erforderlich. Hierfür sind 3 Hauptverfahren prinzipiell geeignet, nämlich 1. die Lokalisierung auf metabolischem Wege, 2. die Lokalisierung auf mikromechanischer Grundlage (mit Hilfe von Kolloiden und Suspensionen) und 3. die Lokalisierung auf technisch-mechanische Weise (prinzipiell ähnlich wie bei Radiumpräparaten). Es werden zahlreiche Beispiele der experimentellen und klinischen Anwendung dieser 3 Hauptmethoden demonstriert und die vorläufigen therapeutischen Resultate mitgeteilt, welche ermutigend sind. Mehrere Originalverfahren des Vortragenden haben bereits Schule gemacht. Es werden insbesondere die verschiedenartigen Anwendungen der Isotope Radiojod, Radiophosphor, Radiozink, Radiogallium, Radiogold und Radiokobalt be sprochen.

$$
\text { Sitzung vom 28. Mai } 1954 .
$$

AUTRUM, H. J.: Nene Beiträge zur Physiologie des Sehens. Schon lange sind entoptische Erscheinungen beim
Menschen bekannt; ihre konsequente Auswertung führt zu dem Schluß, daß die optische Wahrnehmung die Bewegung des Bildes auf der Retina zur Voraussetzung hat; wird das Bild relativ zur Retina nicht bewegt, so verschwindet es durch Lokaladaptation. Vergleichende Untersuchungen ergeben ähnliche Grundprinzipien für das Sehen der Insekten, bei denen die Fähigkeit, bewegte retinale Bilder zu perzipieren, extrem gesteigert ist. Die elektrophysiologischen und physiologisehchemischen Voraussetzungen dieser zeitlichen Analyse optischer Erscheinungen werden dargestellt.

\section{Sitzung vom 25. Juni 1954.}

KEPP, R. K., D. HOFMANN, G. OEHLERT und H. W. VASTERLING: Die Wirkungsmechanismen des Cysteins be seiner Anwendung im aktiven Strahlensehntz. Ausgehend von der Tatsache, daß die Wirkmng ionisierender Strahlungen in biologischen Geweben in komplexer Weise auf einer Reihe von Elementarprozessen, der "Trefferwirkung", der ,indirekten Strahlenwirkung ${ }^{6}$ und der ,weitreichenden indirekten Strahlenwirkung", sowie auf eine Vielzahl won Prozessen höherer Ordnung beruht, die zur Manifestation der ,all gemeinen Strahlenwirkung" führen, wird die Frage untersucht, ob das Cystein bei seiner Wirkung im aktiven Strahlen. schutz an einem, mehreren, oder sämtlichen der genannten Prozesse anzugreifen vermag. Aus der umfangreichen vorliegenden Literatur und ans zahlreiohen eigenen Unter suchungsergebnissen läßt sich eine ebenso komplexe Wirkungsweise des Cysteins folgern, wie sie der Strahlenwirkung selbst zukommt. Uber die für eine Anwendung des Cysteins bei der Strahlenbehandlung bösartiger Tumoren notwendige Voraus. setzung einer günstigen Tumorselektivität der Cysteinwirkung kann nooh nichts ansgesagt werden.

\section{Sitzung vom 9. Juli 1954.}

HARBERS, E.: Isotopenumtersuchungen zum Stofiwechsel der Nucleinsäuren. Nach Schilderung neuer Kenntnisse zur Chemje der Nucleinsäuren wird die Bedeutung der Desoxyribonucleinsäure (DNS) als chemisches Substrat des genetischen. Materials der Zelle diskutiert. Die mit Radioisotopen durchgeführten Stoffweohseluntersuchungen der Nucleinsäuren werden vor allem bezüglich der Beziehungen zwischen DNS.Neubildung and Wachstumsrate behandelt. An Hand eigener Versuche und der Ergebnisse anderer Autoren wird gezeigt, daß die DNS offenbar über die Erfordernisse der Zellteilung hinaus vom Gewebe synthetisiert wird, daß also auch das genetische Material der Zelle einer Dynamik im Sinne von Auf- und Abbau unterliegt. Weiter wird über die Hemmung der DNS-Neubildung durch Röntgenstrahlen sowie die Strahlendosisabhängigkeit dieses Effektes berichtet und abschließend auf die Bedeutung neuer Methoden hingewiesen, die eine Zerlegung der DNS in mehrere heterogene Fraktionen erlauben. Dazu wurden einige eigene Befunde vorgelegt, die mit einer solchen Methode erhoben wurden.

R. K. KEPP (Göttingen).

\title{
TAGESGESCHICHTE.
}

Hochschulnachrichten.

Berlin. Freie Universität. Prof. Dr. R. LINK wurde auf den Lehrstuhl für Hals-Nasen-Ohrenheilkunde berufen. Habilitiert wurden Dr. J.H. Clemens (Anatomie), Dr. H. Penzholz (Neurochirurgie) und Dr. F. STEIN (Allgemeine Pathologie und Pathologische Anatomie).

Ditsseldorf. Prof. Dr. F. Grosse-Brock horf (Tnnere Medizin), Bonn, wurde zum Direktor der I. Medizinisohen Klinik in Düsseldorf ernannt.

Gießen. Prof. Dr. R. M. BoHnstedT (Dermatologie) wurde zum Dekan der Medizinischen Fakultät gewählt.

Lausanne. Dem Ordinarius für Physiologie an der Universität Lausanne, Prof. Dr. A. FubiscH, ist der Marcel Benoist Preis zuerkannt worden anf Grund seiner Monographie: ,Nouvelles méthodes d'étude des échanges gazeux et de la fonction pulmonaire."

München. Prof. Dr. Dr. J, HeIss (Jena) wurde auf den o. Lehrstuhl für Zahnheilkunde an die Universität München berufen; Prof. Dr. H. vaN THTet (Köln), hat den Ruf auf den Lehrstuhl für Zahnprotetik angenommen. $\mathrm{Zu}$ Privatidozenten wurden ernannt Dr. A. Strupprise (Innere
Medizin), Dr. Tr. HellarÜGGE (Pädiatrie) und Dr. J.-E Mexer (Psychiatrie und Neurologie). Prof. Dr. Dr. P. P. Kranz (Zahnheilkunde) feierte am 29. 12.54 seinen 70. Geburtstag.

Münster. Doz. Dr. H. PAU (Ophthalmologie), wurde von der medizinischen Akademie Düsseldorf an die Universität Münster umhabilitiert.

Die "Gesellschatt für arbeitswissenschattliche Forschung e.V." veranstaltet vom 17.-19. März 1955 ihren 2. Arbeitswissenschaftlichen Kongre $B$ in Dortmund. Rahmenthema: „Der altere Mensch in der Arbeit und im Betrieb" "Auskunft durch Dr. H. KELLNER, München 8, Schneckenburgerstr. 41 .

Der Lady Tata Memorial Fund gewährt Beihilfen und Stipendien für die Erforschung von Blutkrankheiten, insbesondere der Leukämie. Anträge müssen bis 31. 3. 1955 eingegangen sein. Antragsformulare durch: The Secretary of the Seientifie Committee, Lady Tata Memorial Trust, 38 old Queen Street, London, S.W.1.

$$
\text { Berichtigung. }
$$

Klin. Wsohr. 1954, XII. Im Inhaltsverzeichnis muB der Name WrTzLra heißen, anstatt WrrzLER (vgl. die Arbeit. WrTzleb, Gollwitzen-Mirme und DoNat). 